\title{
Magnetic resonance imaging parameters of the greater tuberosity morphology in different types of chronic rotator cuff tears
}

\author{
Anqi Wang \\ Peking University First Hospital \\ Hongzhang Lu ( $\square$ wangaqonline@163.com ) \\ Peking University First Hospital
}

\section{Research article}

Keywords: chronic rotator cuff tear, bursal-side rotator cuff tears, articular-side rotator cuff tears, fullthickness rotator cuff tears, greater tuberosity, magnetic resonance imaging

Posted Date: March 30th, 2020

DOI: https://doi.org/10.21203/rs.3.rs-19791/v1

License: (c) (i) This work is licensed under a Creative Commons Attribution 4.0 International License.

Read Full License 


\section{Abstract}

Background: The purpose of this study was to explore the distinction and relation among different types of chronic rotator cuff tears, in terms of some magnetic resonance imaging (MRI) parameters that reflected the greater tuberosity morphology.

Methods: This study retrospectively assessed 160 patients diagnosedwith chronic rotator cuff tears (including bursal-side, articular-side and full-thickness rotator cuff tears) and 48 controls diagnosed without rotator cuff tears by MRI. The types of rotator cuff tear were confirmed by shoulder MRI and arthroscopy. Oblique coronal sections of MRI were used to measure the parameters including greater tuberosity abduction angle(GTAA), greater tuberosity width index (GTWI), and greater tuberosity height index(GTHI).

Results: GTAA in patients with articular-side and full-thicknessrotator cuff tearswere smaller than those in patients with bursal-side rotator cuff tears and in controls.GTWI and GTHI were present in greater proportions in patients with articular-side and full-thickness rotator cuff tearsthan in patients with bursalside rotator cuff tears and in controls. There was no significant difference between the articular-side and full-thickness cuff tears regarding GTAA, GTWI, or GTHI. The same was true between bursal-side rotator cuff tears and the controls.

Conclusions: The greater tuberosity abduction angles are smaller, and greater tuberosity are more convex outward and upwardin patients with articular-side or full-thickness rotator cuff tears, than those in patients with bursal-side rotator cuff tears or in controls.

\section{Introduction}

Rotator cuff tear, resulting from various causes that lead to integrity violation of the supraspinatus, infraspinatus, teres minor, or subscapular tendon, is a common dysfunction of the shoulder joint. The incidence of the rotator cuff tear in population is approximately $20.7 \%-22.1 \%$, and supraspinatus tendon tear is most common[1,2]. The incidence of chronic rotator cuff tear increases with age[3]. Rotator cuff tear can be divided into full-thickness tear and partial-thickness tear, and the latter includes bursal-side, articular-side and intratendinous tear $[4,5]$. The pathogenesis of chronic rotator cuff tear is the result of the combination of both intrinsic and extrinsic causes. The intrinsic causes include age-related metabolic and vascular degeneration in rotator cuff. Extrinsic causes include subacromial impingement, chronic and repetitive traumatic events[6-8]. From the anatomical perspective, bursal-side cuff tears are often associated with subacromial impingement $[9,10]$, and articular-side cuff tears are related to degeneration and stress imbalance in the rotator cuff tendons $[11,12]$. The causes of intratendinous tear remain controversial, but the majority view is differential shear stress within the tendon leads to it [13-15].

The available studies corroborate the idea that the lateral acromion angle (LAA) which reflects the glenoid inclination, and the acromial index (AI) which quantifies the acromial coverage, are larger in patients with full-thickness cuff tears than in controls without rotator cuff tears[16-22]. However, some other studies 
have indicated that the pathogenesis of partial-thickness rotator cuff tears has no correlation with LAA or $\mathrm{Al}$, and no difference has been observed between different types of chronic rotator cuff tears[20].

Footprints of supraspinatus, infraspinatus, and teres minor tendons are distributed around the greater tuberosity, which is close to the position of the acromion. Several researchers have proposed that bone mineral density decrease and cystic degeneration of the greater tuberosity are risk factors for chronic rotator cuff tears [23-27]. However, the roles of the greater tuberosity morphology in different types of chronic rotator cuff tears have not been investigated to date. MRI can assess shoulder joints for several research subjects in the same neutral position. Oblique coronal sections of MRI can be used to assess several parameters that reflect the greater tuberosity morphology. We used MRI to explore the distinctions and relations of the greater tuberosity morphology in bursal-side, articular-side and full-thickness supraspinatus tendon tears. This study may provide the surgeon with a new perspective in differential diagnosis on different types of chronic rotator cuff tears.

\section{Materials And Methods}

\section{Research subjects}

160 patients diagnosed with chronic rotator cuff tears, including bursal-side rotator cuff tears (group A), articular-side rotator cuff tears (group B) and full-thickness rotator cuff tears (group C), from July 2015 to March 2019 in Peking University First Hospital were enrolled in the study. The inclusion criteria were as follows: patients who 1) underwent persistent shoulder pain and disability symptoms; 2) showed ineffective response to 6 months or more conservative treatment and received shoulder arthroscopic surgery; 3 ) diagnosed with supraspinatus tendon tears based on MRI and surgical findings. In addition, 48 controls (group D) who underwent health examination and diagnosed without rotator cuff tears according to MRI were enrolled in the study. The exclusion criteria were as follows: patients who 1) had congenital shoulder instability; 2) shoulder surgical history; 3 ) acute trauma; 4) vascular and metabolic changes due to such factors as diabetes, rheumatoid arthritis and other medical diseases; 5) obvious retraction of torn rotator cuff tendons and fatty atrophy of the rotator cuff according to MRI.

This study was approved by the Human Subjects Institutional Review Board at Peking University First Hospital. Patient outcomes were collected independently from participants with informed consent.

\section{MRI parameters assessments}

Two independent readers, who were all blinded to the patients' diagnoses, assessed all the MRI parameters. Standardized MRI of shoulders with the arm in a neutral position were used to assess the parameters of the greater tuberosity including 1 ) the greater tuberosity abduction angle (GTAA; Fig. 1A), which reflected the greater tuberosity abduction degree when the articular side of supraspinatus tendon was pinched against the posterior-superior glenoid rim; 2) the greater tuberosity width index (GTWl; Fig. 1B), which reflected the width of the greater tuberosity morphology; 3 ) and the greater tuberosity height index (GTHI, Fig. 1C), which reflected the height of greater tuberosity morphology. 


\section{Statistics}

Data were analyzed by Statistical package for program (Version12, SPSS, Chicago, IL). One-way ANOVA followed by Bonferroni post hoc test was used to compare the difference of continuous variables between the groups, and the statistic was demonstrated as mean \pm SD. Chi-square test was used to compare the difference of dichotomous variables between the groups. These two tests were used to compare the patient demographic data and MRI parameters between the groups. $P<0.05$ was regarded as statistical significance.

\section{Results}

\section{Patient demographics and characteristics}

Regarding preoperative demographic data and characteristics, there was no statistical difference between the groups regarding age, gender and side (all $P>0.05$, Table 1 ).

\section{MRI parameters of the greater tuberosity}

MRI parameters including GTAA, GTWI and GTHI in 4 groups were demonstrated (Table 2).

In the GTAA (greater tuberosity abduction angle, Fig. 2A), there was significant difference in the GTAA between the 4 groups $(p<0.001)$. The GTAA of Group D were greater than that of Group B $(p=0.001)$ and Group C $(p=0.005)$, also the GTAA of Group A were greater than that of Group B $(p<0.001)$ and Group C $(p=0.001)$. There was no significant difference between Groups $D$ and $A(p=0.812)$, also between Groups B and C ( $p=0.085)$.

Regarding the GTWI (greater tuberosity width index, Fig. 2B), there was significant difference between the 4 groups $(p<0.001)$. The value for Group $D$ was less than that for group $B(p<0.001)$ and group $C(p<$ $0.001)$, but there was no significant difference $(p=0.196)$ from group $A$. Group A was smaller than group $B(p=0.004)$ and group $C(p=0.005)$, and there was no significant difference between group $B$ and $C(p=$ $0.498)$.

The GTHI (greater tuberosity height index, Fig. 2C) exhibited a significant difference between the 4 groups $(p=0.001)$. The GTHI of Group D were smaller than that of group $B(p=0.013)$ and group $C(p=0.022)$, and there was no significant difference from group $A(p=0.279)$. The GTHI of Group A were smaller than that of group $B(p=0.001)$ and group $C(p=0.005)$, and there was no significant difference between group $B$ and $C(p=0.942)$.

\section{Discussion}

Chronic rotator cuff tears, which affect the patients' quality of life, lead to global burden with severe health-care and socioeconomic consequences. Many studies have demonstrated that several anatomical factors of acromion and greater tuberosity are associated with chronic rotator cuff tears. Kanatli $U$ et al. 
[16] and Balke $M$ et al.[17] have indicated that on shoulder $X$ rays, the lateral acromion angle (LAA), which reflects the glenoid inclinations of patients with full-thickness rotator cuff tears, is significantly higher than that in controls without rotator cuff tears. Moor BK et al.[19] and Nyffeler RW et al. [21] have demonstrated that the acromion index (Al), which quantifies the acromial coverage exhibits the same results mentioned above. In contrast, some other researchers have found that the inclination and extension of the acromion are not correlated with the morbidity of partial rotator cuff tears, and no significant differences among different types of chronic rotator cuff tears[20]. Most footprints of the rotator cuff tendons are distributed around the greater tuberosity, which is close to the position of the acromion, so the characteristics of greater tuberosity may distribute to chronic rotator cuff tears. Previous studies have reported that the reduction in bone density of the greater tuberosity leads to decrease in strength of the rotator cuff attachment, which causes chronic rotator cuff tears[23]. Cystic changes in the front of greater tuberosity are also closely related to rotator cuff tears[27]. However, the relationship between the morphological features of greater tuberosity and chronic rotator cuff tears is unclear. Similar to previous research on the morphological characteristics of acromion, we selected the parameters that reflect greater tuberosity morphology including abduction angle, width and height, to determine their relations with different types of chronic rotator cuff tears.

Although most previous studies have measured the morphological parameters of acromion from shoulder $X$ rays, initially the LAA and Al were measured on the oblique coronal plane of MRI[28, 29]. In this study, we also measured the morphological parameters of greater tuberosity on the oblique coronal plane of shoulder MRI, which enabled standardized and accurate evaluation among different research objects under the same position and in different scanning planes.

The GTAA measured in this research is a dynamic parameter that could reflect the abduction degree of greater tuberosity when the articular side of supraspinatus tendon is pinched against the posteriorsuperior glenoid rim. According to the GTAA analysis, we found that the GTAA of patients with articularside and full-thickness rotator cuff tears was significantly less than that of the controls, whereas the GTAA of patients with bursal-side rotator cuff tears was not obviously different from that of the controls. This finding suggested that the reduction of the GTAA was a risk factor for articular-side and fullthickness rotator cuff tears and had no significant correlation with bursal-side rotator cuff tears. Supposedly, when the shoulder joint abducts, the greater tuberosity with low GTAA will collide with the posterior-superior glenoid rim more frequently, which gradually leads to articular-side rotator cuff tear. Moreover, the GTAA exhibited no significant difference between the articular-side tear group and the fullthickness tear group, which suggests that the full-thickness rotator cuff tears may originate from articular-side tears.

To study the morphological features of greater tuberosity, except its dynamic parameter GTAA, it is necessary to determine whether the static parameters of greater tuberosity are associated with different types of chronic rotator cuff tears. In this study, the GTWI and GTHI respectively reflected the extents of the greater tuberosity being convex outward and upward in the proximal humerus. The results demonstrated that the greater tuberosity of the patients with chronic articular-side and full-thickness 
rotator cuff tears was more convex outward and upward than that of the patients with bursal-side tears and the controls. The results suggest that increased width and height indices were risk factors for chronic articular-side and full-thickness rotator cuff tears. Moreover, the results from the articular-side tear group exhibited no significant differences from those of the full-thickness tear group, which suggests again that the full-thickness rotator cuff tears may originate from articular-side tears. Based on anatomy, if the greater tuberosity gets widened and heightened due to hyperostosis and osteophyte, when the shoulder abducts, collision and friction between the articular-side of supraspinatus tendon and the posteriorsuperior glenoid rim are increased, gradually result to chronic rotator cuff tears. Simultaneously, the morphological changes of greater tuberosity may be a secondary response to chronic rotator cuff tears. Davidson et al.[30] reported a case of a 23-year-old right-handed professional baseball pitcher with an articular-side rotator cuff tear. An exploration of the pathogenesis revealed that frequent abduction and external rotation led to an articular-side tear of the supraspinatus tendon that pinched against the posterior-superior glenoid rim, which supports our research results and mechanisms.

Our study find that the greater tuberosity abduction angles are smaller, but greater tuberosity are more convex outward and upward in patients with articular-side or full-thickness rotator cuff tears, than in patients with bursal-side rotator cuff tears or in controls. Chronic articular-side and full-thickness rotator cuff tears are related to the morphology of greater tuberosity, which can assist us to estimate the type of chronic rotator cuff tears when we have difficulty in differential diagnosis via shoulder MRI, and to find the supraspinatus tendon's tear position under arthroscopic surgery more quickly.

Certainly, our study has several limitations. Firstly, the number of enrolled patients was small, and the specific duration of disease was not considered. Secondly, chronic rotator cuff tears result from multiple factors. Except for the parameters mentioned in our study, whether other morphological features of the shoulder joint distribute to different types of chronic rotator cuff tears needs to be explored in the future.

\section{Conclusion}

Chronic articular-side and full-thickness rotator cuff tears are related to the morphology of greater tuberosity, which may provide the surgeon with a new perspective in differential diagnosis on different types of chronic rotator cuff tears.

\section{Declarations}

\section{Ethics approval and consent to participate}

Not applicable.

\section{Consent for publication}

All authors read the final manuscript and approved for publication. 
Availability of data and material

All data generated or analyzed during this study are included in this published article.

\section{Competing interests}

The authors declare that they have no competing interests.

\section{Funding}

Not applicable.

\section{Authors' contributions}

Anqi Wang and Hongzhang Lu designed the study and assess the MRI parameters. Anqi Wang collected patient demographic data. All authors analyzed the data and wrote the paper. All authors read and approved the final manuscript.

\section{Acknowledgements}

Not applicable.

\section{References}

1. H. Minagawa, N. Yamamoto, H. Abe, M. Fukuda, N. Seki, K. Kikuchi, H. Kijima, E. Itoi, Prevalence of symptomatic and asymptomatic rotator cuff tears in the general population: From mass-screening in one village, J Orthop, 10 (2013) 8-12.

2. A. Yamamoto, K. Takagishi, T. Osawa, T. Yanagawa, D. Nakajima, H. Shitara, T. Kobayashi, Prevalence and risk factors of a rotator cuff tear in the general population, J Shoulder Elbow Surg, 19 (2010) 116-120.

3. A.A. Sayampanathan, T.H. Andrew, Systematic review on risk factors of rotator cuff tears, J Orthop Surg (Hong Kong), 25 (2017) 2309499016684318.

4. O.R. McConville, J.P. lannotti, Partial-thickness tears of the rotator cuff: evaluation and management, J Am Acad Orthop Surg, 7 (1999) 32-43.

5. E.J. Strauss, M.J. Salata, J. Kercher, J.U. Barker, K. McGill, B.R. Bach, Jr., A.A. Romeo, N.N. Verma, Multimedia article. The arthroscopic management of partial-thickness rotator cuff tears: a systematic review of the literature, Arthroscopy, 27 (2011) 568-580.

6. R.P. Finnan, L.A. Crosby, Partial-thickness rotator cuff tears, J Shoulder Elbow Surg, 19 (2010) 609616.

7. S. Mehta, J.A. Gimbel, L.J. Soslowsky, Etiologic and pathogenetic factors for rotator cuff tendinopathy, Clin Sports Med, 22 (2003) 791-812. 
8. J. Ozaki, S. Fujimoto, Y. Nakagawa, K. Masuhara, S. Tamai, Tears of the rotator cuff of the shoulder associated with pathological changes in the acromion. A study in cadavera, J Bone Joint Surg Am, 70 (1988) 1224-1230.

9. C.S. Neer, 2nd, Anterior acromioplasty for the chronic impingement syndrome in the shoulder: a preliminary report, J Bone Joint Surg Am, 54 (1972) 41-50.

10. C.S. Neer, 2nd, Impingement lesions, Clin Orthop Relat Res, (1983) 70-77.

11. K.M. Shin, Partial-thickness rotator cuff tears, Korean J Pain, 24 (2011) 69-73.

12. R.W. Nyffeler, D.C. Meyer, Acromion and glenoid shape: Why are they important predictive factors for the future of our shoulders?, EFORT Open Rev, 2 (2017) 141-150.

13. S.B. Lee, T. Nakajima, Z.P. Luo, M.E. Zobitz, Y.W. Chang, K.N. An, The bursal and articular sides of the supraspinatus tendon have a different compressive stiffness, Clin Biomech (Bristol, Avon), 15 (2000) 241-247.

14. K. Yamanaka, T. Matsumoto, The joint side tear of the rotator cuff. A followup study by arthrography, Clin Orthop Relat Res, (1994) 68-73.

15. P. Clavert, Y. Le Coniat, J.F. Kempf, G. Walch, Intratendinous rupture of the supraspinatus: anatomical and functional results of 24 operative cases, Eur J Orthop Surg Traumatol, 26 (2016) 133-138.

16. U. Kanatli, T. Ayanoglu, E. Aktas, M.B. Ataoglu, M. Ozer, M. Cetinkaya, Grade of coracoacromial ligament degeneration as a predictive factor for impingement syndrome and type of partial rotator cuff tear, J Shoulder Elbow Surg, 25 (2016) 1824-1828.

17. M. Balke, C. Schmidt, N. Dedy, M. Banerjee, B. Bouillon, D. Liem, Correlation of acromial morphology with impingement syndrome and rotator cuff tears, Acta Orthop, 84 (2013) 178-183.

18. D. Musil, P. Sadovsky, M. Rost, J. Stehlik, L. Filip, [Relationship of acromial morphology and rotator cuff tears], Acta Chir Orthop Traumatol Cech, 79 (2012) 238-242.

19. B.K. Moor, K. Wieser, K. Slankamenac, C. Gerber, S. Bouaicha, Relationship of individual scapular anatomy and degenerative rotator cuff tears, J Shoulder Elbow Surg, 23 (2014) 536-541.

20. V. Pandey, D. Vijayan, S. Tapashetti, L. Agarwal, A. Kamath, K. Acharya, S. Maddukuri, W.J. Willems, Does scapular morphology affect the integrity of the rotator cuff?, J Shoulder Elbow Surg, 25 (2016) 413-421.

21. R.W. Nyffeler, C.M. Werner, A. Sukthankar, M.R. Schmid, C. Gerber, Association of a large lateral extension of the acromion with rotator cuff tears, J Bone Joint Surg Am, 88 (2006) 800-805.

22. J.R. Kim, K.J. Ryu, I.T. Hong, B.K. Kim, J.H. Kim, Can a high acromion index predict rotator cuff tears?, Int Orthop, 36 (2012) 1019-1024.

23. M.J. Tingart, M. Apreleva, D. Zurakowski, J.J. Warner, Pullout strength of suture anchors used in rotator cuff repair, J Bone Joint Surg Am, 85 (2003) 2190-2198.

24. Y. Sakamoto, A. Kido, K. Inoue, G. Sakurai, T. Hashiuchi, M. Munemoto, Y. Tanaka, In vivo microstructural analysis of the humeral greater tuberosity in patients with rotator cuff tears using multidetector row computed tomography, BMC Musculoskelet Disord, 15 (2014) 351. 
25. L.B. Fritz, H.A. Ouellette, T.A. O'Hanley, A. Kassarjian, W.E. Palmer, Cystic changes at supraspinatus and infraspinatus tendon insertion sites: association with age and rotator cuff disorders in 238 patients, Radiology, 244 (2007) 239-248.

26. C. Ruotolo, J.E. Fow, W.M. Nottage, The supraspinatus footprint: an anatomic study of the supraspinatus insertion, Arthroscopy, 20 (2004) 246-249.

27. F. Suluova, U. Kanatli, B.Y. Ozturk, E. Esen, S. Bolukbasi, Humeral head cysts: association with rotator cuff tears and age, Eur J Orthop Surg Traumatol, 24 (2014) 733-739.

28. M.P. Banas, R.J. Miller, S. Totterman, Relationship between the lateral acromion angle and rotator cuff disease, J Shoulder Elbow Surg, 4 (1995) 454-461.

29. P. Tetreault, A. Krueger, D. Zurakowski, C. Gerber, Glenoid version and rotator cuff tears, J Orthop Res, 22 (2004) 202-207.

30. P.A. Davidson, N.S. Elattrache, C.M. Jobe, F.W. Jobe, Rotator cuff and posterior-superior glenoid labrum injury associated with increased glenohumeral motion: a new site of impingement, $\mathrm{J}$ Shoulder Elbow Surg, 4 (1995) 384-390.

\section{Tables}

Due to technical limitations, the tables are only available as a download in the supplemental files section.

\section{Figures}

\section{Figure 1}

Overview of the MRI parameters that reflected greater tuberosity morphology (assessed on the plane where the distance from the inferior border to the superior border of the glenoid fossa was the longest). (A) The GTAA, greater tuberosity abduction angle, was measured between a first line connecting the inferior border to the superior border of the glenoid fossa and a second line connecting the inferior border of the glenoid to the most inferomedial point of the greater tuberosity. (B) The GTWI, greater tuberosity width index, was determined by the ratio of the distance from L1 (the lateral aspect of the greater tuberosity) to L2 (the medial aspect of the greater tuberosity; LN) with the distance from L1 to L3 (the medial aspect of the humeral head; LM). L1, L2 and L3 were parallel to L0 (the humeral shaft anatomical axis). (C) The GTHI, greater tuberosity height index, was determined by the ratio of the distance from L4 (the inferior plane of the humeral head) to L5 (the superior plane of the greater tuberosity; IT) with the distance from L4 to L6 (the superior plane of the humeral head; IS). L4, L5 and L6 are perpendicular to L0. 

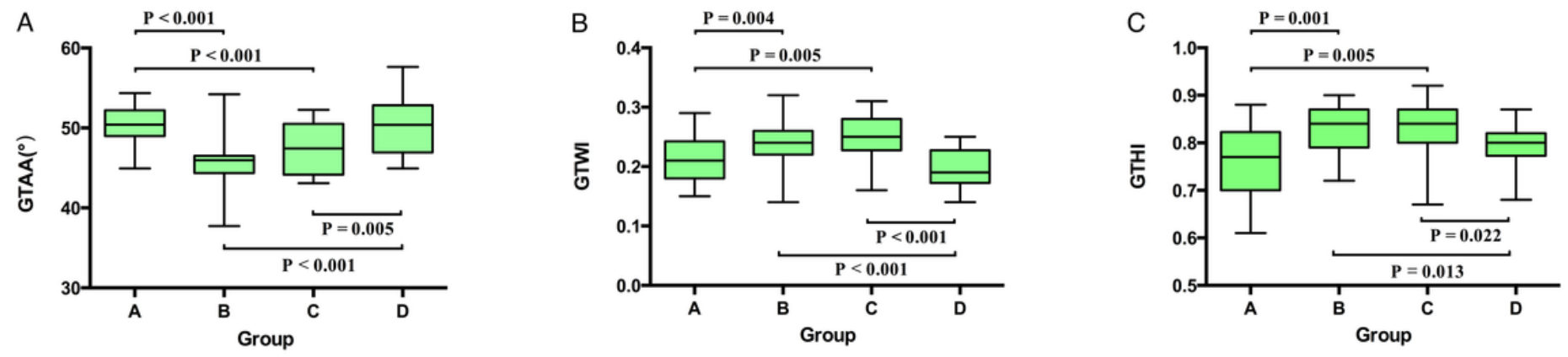

Figure 2

Comparison of GTAA, GTWI and GTHI between the groups. (A) The comparison of GTAA between the groups. (B) The comparison of GTWI between the groups. (C) The comparison of GTHI between the groups.

\section{Supplementary Files}

This is a list of supplementary files associated with this preprint. Click to download.

- Table2.png

- Table1.png 